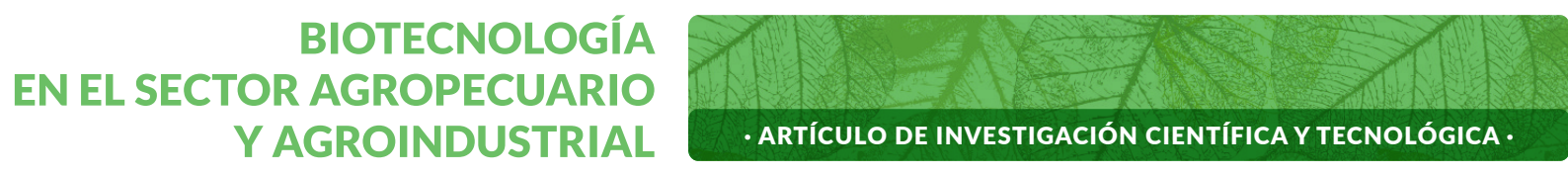

Vol. 16 No 2 · Julio - Diciembre 2018 • ISSN - 1692-3561 · ISSN-e 1909-9959 · doi: http://dx.doi.org/10.18684/bsaa.v16n2.97

\title{
Descripción del ciclo fenológico de cuatro ecotipos de (Chenopodium quinua Willd.), en Puracé - Cauca, Colombia
}

\section{Description of phenological cycle of four ecotypes of (Chenopodium quinoa Willd.), at Puracé - Cauca, Colombia}

\section{Descrição da ciclo fenological de quatro ecótipos quinua (Chenopodium quinoa Willd.), em Puracé - Cauca, Colombia}

CONSUELO MONTES- ROJAS ${ }^{1}$, GUIDO ARY BURBANO-CATUCHE ${ }^{2}$, EDWIN FERNANDO MUÑOZ-CERTUCHE ${ }^{3}$ YIMY CALDERÓN-YONDA $^{4}$

\section{RESUMEN}

En el departamento del Cauca, municipio de Puracé vereda El Acuario, localizado a 3023 m.s.n.m., en el marco del proyecto Cadena productiva de la quinua se evaluó el ciclo fenológico de 4 ecotipos de quinua y su rendimiento con el fin de seleccionar

Recibido para evaluación: 7 de Agosto de 2017.

Aprobado para publicación: 7 de Enero de 2018.

1 Universidad del Cauca, Departamento de Ciencias Agropecuarias, Facultad de Ciencias Agrarias, Grupo de Investigación para el Desarrollo Rural (Tull). M Sc. Profesora de planta. Popayán, Colombia.

2 Fundación de profesionales para el desarrollo integral comunitario PRODESIC. Ingeniero Agropecuario. Popayán, Colombia.

3 Fundación de profesionales para el desarrollo integral comunitario PRODESIC. Ingeniero Agroindustrial. Popayán, Colombia

4 Fundación de profesionales para el desarrollo integral comunitario PRODESIC. Ingeniero Agroindustrial. Popayán, Colombia

Correspondencia: cmontesr@unicauca.edu.co 
y determinar la duración de las etapas del ciclo productivo que determinan su manejo agronómico en alturas superiores a 3000 m.s.n.m. El propósito es buscar una opción para rotación de cultivo de papa que aporte a la seguridad alimentaria regional. Este estudio se realizó durante el 2016 bajo condiciones de campo, en el resguardo indígena de Paletará. Se utilizó un diseño de bloques completos al azar con 3 repeticiones donde los tratamientos fueron los ecotipos, se registraron etapas fenológicas, se evaluó contenido de saponinas y el rendimiento. Se determinó que la quinua presenta dos etapas fenológicas bien marcadas, etapa vegetativa con 6 fases y etapa reproductiva con 7 fases; la duración del ciclo productivo de los 4 ecotipos estuvo entre 154 y 213, permitiendo clasificarlos como semiprecoces y semitardios, el contenido de saponinas estuvo entre 0,005 y 0,012\%. Se concluyó que de los 4 ecotipos evaluados se recomienda aurora por precocidad, sabor dulce y mejor rendimiento.

\section{ABSTRACT}

At Cauca, municipality of Puracé, sidewalk The Aquarium, located at 3023 meters above sea level, within the framework of the Quinoa production chain project, the phenological cycle of 4 quinoa ecotypes and their yield were evaluated in order to select and determine the duration of the stages of the productive cycle that determine their agronomic management at heights greater than 3000 meters above sea level. The purpose is to look for a potato crop rotation option that will contribute to regional food security. This study was carried out during 2016 under field conditions, in the indigenous reservation of Paletará. We used a randomized complete block design with 3 replicates where treatments were ecotypes, phenological stages were recorded, saponin content and performance were evaluated. It was determined that quinoa presents two well marked phenological stages, vegetative stage with 6 phases and reproductive stage with 7 phases; the duration of the productive cycle of the 4 ecotypes was between 154 and 213, allowing to classify them as semiearly and semidelayed, the content of saponins was between 0,005 and 0,012\%. It was concluded that of the 4 ecotypes evaluated, aurora is recommended for precocity, sweet taste and better yield.

\section{RESUMO}

En el departamento do Cauca, município de Puracé vereda El Acuario, localizado a 3023 msnm, no marco do projeto Cadena produtiva da quinua, avaliada pelo ciclo fenológico de 4 ecotipos de quinua e desempenho no final da temporada e determinar a duração das etapas do ciclo produtivo e determinante da gestão agronómica em alturas superiores a $3000 \mathrm{msnm}$ O objetivo é buscar uma opção para rotação de cultivo de papa que aporte a segurança alimentaria regional. Este estudo se realizou durante o 2016 sob condições de campo, na residência indígena de Paletará. Se utilizou um projeto de blocos completos com azar com 3 repetições onde os tratamentos foram os ecotipos, se registraram etapas fenológicas, se avaliou o conteúdo de saponinas e o desempenho. Se determinou que a quinua apresenta duas etapas fenológicas bem marcadas, etapa vegetativa com 6 fases e etapa reprodutiva con 7 fases; a duração do ciclo produtivo dos 4 ecotipos estude
PALABRAS CLAVE:

Quinua, Genotipos, Fenología, Selección, Saponinas, ecotipos.

\section{KEYWORDS:}

Quinoa, Genotypes, Phenology, Selection, Saponins.

\section{PALAVRAS-CHAVE:}

Quinoa, Genotipagem, Fenologia, Selecção, Saponinas. 
entre 154 y 213 , permitendo classificarlos como semiprecoces e semitardios, o conteúdo de saponinas justo entre 0,005 y $0,012 \%$. Se conclui que é de 4 ecotipos avaliados por um atraente e melhor desempenho.

\section{INTRODUCCIÓN}

La quinua (Chenopodium quinoa Willd.) se ha cultivado principalmente en la cordillera de los andes en Bolivia, Perú, Ecuador y Colombia [1], fue uno de los principales alimentos de los pueblos, andinos preincaicos e incaicos, hasta el descubrimiento de América.

Las bondades de la quinua radican en su alto valor nutricional, con un contenido de proteína entre 13,81 y $21,9 \%$ dependiendo de la variedad. Debido al elevado contenido de aminoácidos esenciales de su proteína, la quinua es considerada como el único alimento del reino vegetal que provee todos los aminoácidos esenciales, que se encuentran extremadamente cerca de los estándares de nutrición humana establecidos por la FAO [2]. Se ha encontrado que el balance de los aminoácidos esenciales de la proteína de la quinua es superior al trigo, cebada y soya, comparándose favorablemente con la proteína de la leche [3].

Una característica fundamental de la quinua es que el grano, las hojas y las inflorescencias son fuentes de proteínas de muy buena calidad. La calidad nutricional del grano es importante por su contenido de aminoacidos, siendo rico en lisina y azufrados, mientras que las proteínas de los cereales son deficientes en estos aminoácidos.

A pesar de su buen contenido de nutrientes, las investigaciones realizadas concluyen que los aminoácidos de la proteína, en la harina cruda y sin lavar no están del todo disponibles, porque contienen sustancias que interfieren con la utilización biológica de los nutrientes. Estas sustancias son los glucósidos denominados saponinas [4].

Con base en lo anteriormente expuesto y dada la situación de desnutrición que tiene Colombia en algunos departamentos, la quinua se constituye en un cultivo estratégico para contribuir a la seguridad y soberanía alimentaria debido a su calidad nutritiva, su amplia variabilidad genética, su adaptabilidad y su bajo costo de producción.
En Colombia la quinua se cultiva en los departamentos de Cundinamarca, Boyacá, Nariño y Cauca. En este último, el cultivo se concentra principalmente en la región del Macizo Colombiano, en los municipios de Santa Rosa, Bolívar, Almaguer, La Vega y Rosas y otros municipios del departamento con condiciones climáticas diferentes como: Sotará, Puracé, Toribío, Totoró, Silvia, Caldono y Jámbalo, donde generalmente se siembra el ecotipo conocido como blanca de Jericó, que tiene características favorables para la agroindustria, pero presenta gran variabilidad en su comportamiento, en campo.

Las quinuas cultivadas tienen una gran diversidad genética, mostrando variabilidad en la coloración de la planta, inflorescencia y semilla, en los tipos de inflorescencia, y en el contenido de proteína, saponina y betacianina en las hojas, con lo que se obtiene una amplia adaptación a diferentes condiciones agroecológicas (suelos, precipitación, temperatura, altitud, resistencia a heladas, sequía, salinidad o acidez) [5].

Con el objeto de seleccionar al menos un ecotipo de quinua para condiciones de más de 3000 m.s.n.m. y que sea una opción para rotación de cultivo de papa y aporte a la seguridad alimentaria regional este trabajo se propuso evaluar el ciclo fenológico de 4 ecotipos de quinua en el municipio de Puracé.

\section{MÉTODO}

En el municipio de Puracé, vereda El Acuario, de propiedad del resguardo indígena de Paletará se seleccionó un lote con manejo convencional, localizado a 3023 m.s.n.m., latitud norte es $-2^{\circ}, 15$ ', $19.5^{\prime \prime}$ y longitud oeste de -760, 30', 09.0".

Los tratamientos estuvieron determinados por los ecotipos y se utilizó un diseño de bloques completos al azar con 3 repeticiones.

\section{Descripción de los ecotipos}

Tunkahuan. Plantas con alturas entre 160 y 230 cm; panojas grandes, bien ramificadas, poco compactas, variando el color de purpura en pleno desarrollo a purpura rosado en formación de grano y purpura anaranjado en inicio de madurez, dejando expuestos los granos blancos y grandes, con diámetro mayor a 2 $\mathrm{mm}$, este ecotipo presenta tolerancia al desgrane [6]. 
Aurora. Plantas con altura que oscila entre 90 y $130 \mathrm{~cm}$; panojas con tonalidad blanca-rosada, ramas pequeñas y flores ubicadas en forma semicompacta; los granos quedan descubiertos cuando maduran y son de color blanco, pequeños, con un diámetro menor a $2 \mathrm{~cm}$ [6].

Blanca de Jericó. Hábito erecto, altura entre 120 y 210 $\mathrm{cm}$. Follaje y panoja de color verde, con bastante ramificación, presenta panoja secundaria, tiene grano de color blanco, de sabor dulce, por lo que no requiere lavado para su posterior consumo o comercialización [6].

Punto rojo. Similar a Tunkahuan en cuanto a longitud de plantas y tamaño de panojas, las cuales, aunque amarantiformes, son semicompactas por mayor concentración de glomérulos, con tolerancia al desgrane, con coloración purpura rojiza en la madurez. Las semillas son blancas, planas y grandes, impregnadas de un polvillo rosado (oxalatos) cuando maduran. Tiene buena tolerancia a mildeo y manchas foliares, aunque es moderadamente susceptible a la mancha ojival de los tallos [6].

\section{Preparación del terreno}

La parcela útil estuvo constituida por los 3 surcos centrales tomando $4 \mathrm{~m}$ efectivos, se dejaron $50 \mathrm{~cm}$ de cabecera a cada lado y los dos surcos laterales como bordes, para tener el efecto de competencia real en campo.

Al momento de siembra se aplicó un mejorador orgánico enriquecido con microorganismos, para mejorar las propiedades físicas, químicas y biológicas del suelo, con dosis de $500 \mathrm{Kg} / \mathrm{ha}$, debido a que la quinua responde muy bien a la aplicación de materia orgánica y su déficit afecta su desarrollo, crecimiento y producción. Adicionalmente, se aplicaron $400 \mathrm{Kg} /$ ha de cal dolomítica para evitar corrección de $\mathrm{pH}$ del suelo y para hacer disponible los nutrientes que hay en el suelo para la planta.

Se aplicaron $80 \mathrm{Kg} / \mathrm{ha}$ de un fertilizante fosfatado enriquecido con Calcio, Magnesio, Silicio y micronutrientes; nitratos en dosis de $100 \mathrm{Kg} / \mathrm{ha}$ con concentraciones de nitrógeno ureico y amoniacal de al menos el $40 \%$ cada uno; una vez las plantas tuvieron hojas verdaderas se iniciaron aplicaciones semanales de elementos menores en forma foliar en dosis de $2 \mathrm{~L} / \mathrm{ha}$.

30 días después de emergencia se realizó reabone al suelo con 22-3-20 y semanalmente hasta el día 90 después de emergencia se aplicaron $2 \mathrm{~L} / \mathrm{ha}$ de fertilizante orgánico foliar; luego en dosis de $2 \mathrm{~L} / \mathrm{ha}$ se hizo suplementación con elementos menores enriquecidos con boro, calcio, fósforo y potasio.

Teniendo en cuenta que el lote provenía de pasto y poseía montículos por el trasegar del ganado, la preparación del terreno fue mecanizada con tractor, se realizó doble arada y pasada con rotavitor para dejar el suelo suelto, tal y como lo requiere el cultivo de quinua.

Una vez preparado el terreno se elaboraron las camas de siembra a distancias de $80 \mathrm{cms}$ entre surcos dejando calles de $1 \mathrm{~m}$ entre parcelas. En total se establecieron 12 parcelas de $5 \mathrm{~m}$ de largo por $3 \mathrm{~m}$ de ancho, con siembra a chorrillo.

Manejo y evaluación. En los primeros 10 días se identificó trozador (Agrotis de privata W.) y babosas, para lo cual se colocaron cebos con el fin de controlar el ataque.

El cebo se preparó mezclando uniformemente $10 \mathrm{~kg}$ de aserrín, $4 \mathrm{~kg}$ de melaza, $4 \mathrm{~kg}$ de clorpirifos y $5 \mathrm{~L}$ de agua. Se aplicó en el perímetro del lote y por las calles. Para controlar la babosa se utilizaron $2 \mathrm{~kg}$ de mata babosa, la cual, se aplicó en los extremos y calles donde se detectó su presencia.

Un mes después de siembra fue necesario aplicar Carbendazim y Metelaxyl en dosis de 0,4 L/ha y 300 $\mathrm{g} / \mathrm{ha}$ respectivamente, para evitar pudriciones, por exceso de humedad.

Labores culturales. Se hizo descapotado, picado, raleo y aporque, con el fin de permitir el óptimo desarrollo de las plantas y evitar estrés por competencia.

El Descapotado se realizó con rastrillo, para romper la corteza que se forma en el suelo y facilitar la emergencia de plántulas; picado, se realizó para eliminar arvenses, remover el suelo y activar los microorganismos del suelo; aporque, para mejorar el anclaje de las plantas y el suministro de nutrientes; raleo, se realizó con el fin de controlar la densidad de siembra determinada en el ensayo.

Se realizó manejo de conservación de suelos, dejando cubierta vegetal para evitar pérdida de suelo por vientos y mejorar la humedad del lote en general. Esta actividad implico hacer corte y control de arvenses con guadaña, dejando el corte sobre las calles. 
Cosecha. La cosecha se realizó en forma manual, cosechando $3 \mathrm{~m}^{2}$ por parcela en forma aleatoria dentro de la parcela útil de muestreo.

\section{Ciclo fenológico}

Para evaluar el ciclo fenológico se registraron los cambios externos visibles del proceso de desarrollo de la planta, La quinua presenta fases fenológicas bien marcadas y diferenciables, las cuales permiten identificar los cambios que ocurren durante el desarrollo de la planta, Se evaluó días a germinación, días a emergencia, aparición de primera hoja verdadera, días a formación de 4 hojas verdaderas, días a formación de 6 hojas verdaderas, aparición de inflorescencia, días a formación de panoja, días a floración, dos estados de grano, lechoso, pastoso y madurez fisiológica.

Para evaluar la germinación de semilla, se colocaron 50 semillas por ecotipo en papel toalla previamente humedecido, posteriormente se ubicaron los paquetes en bolsa plástica para iniciar evaluación a las 24 horas.

\section{Determinación contenido de saponinas}

La quinua presenta un factor anti nutricional, el contenido de saponinas, sustancias amargas que tienen la propiedad de hemolizar los glóbulos rojos y son altamente tóxicas para animales de sangre fría, razón por la cual, se determinó el contenido de saponinas en los 4 ecotipos evaluados.

Se utilizó el método de medición de la espuma (Afro simétrico) y se basa en que las saponinas disueltas en agua y agitadas forman una espuma estable, la altura de esta espuma se correlaciona con el contenido de saponinas en granos de quinua [6].

Se tomaron 0,5 g de granos enteros de quinua y se colocaron en tubos de ensayo, luego se añadieron 5 $\mathrm{mL}$ de agua destilada y se taparon, posteriormente se sacudió vigorosamente cada tubo durante 30 segundos y se dejaron en reposo durante 30 minutos.
Posteriormente, se sacudieron otra vez durante 20 segundos y se dejaron en reposo durante 30 minutos; se sacudieron nuevamente durante 30 segundos y se dio a los tubos una última sacudida como la que se le hace al termómetro oral; se dejó el tubo en reposo durante 5 minutos y luego se midió la altura de la espuma, al 0,1 cm más cercano (Figura 1).

Para calcular el contenido de saponina se utilizaron las siguientes fórmulas: (Ec. 1) (Ec. 2)

\section{RESULTADOS}

Para establecer el ciclo fenológico de cada ecotipo se obtuvieron los promedios de los días para cada fase evaluada, entre parcelas de cada ecotipo.

\section{Fenología de los ecotipos}

Esta evaluación permitió reconocer los cambios en el crecimiento y desarrollo de las plantas como resultado de los factores ambientales y su componente genético, lo que ayuda a determinar los requerimientos nutricionales, edáficos y de manejo para el cultivo en general.

Se determinó que la quinua presenta dos etapas fenológicas bien marcadas, etapa vegetativa que incluye

Figura 1. Altura de la espuma para calcular saponinas.

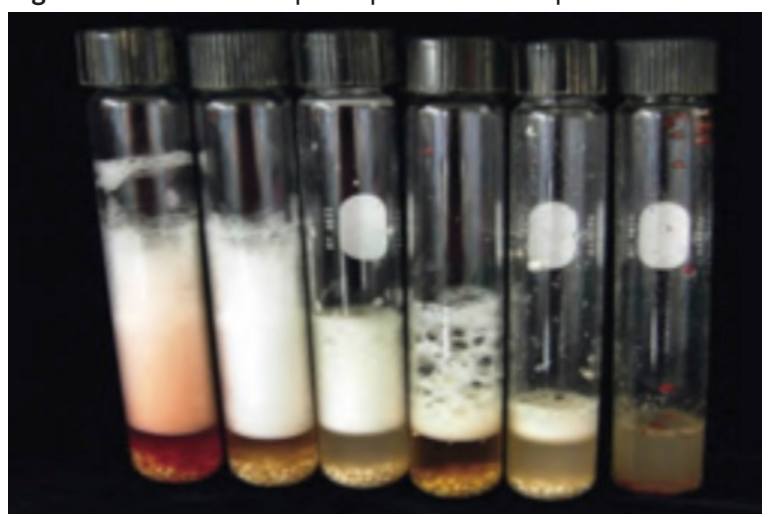

$$
\begin{gathered}
m g \text { de } \frac{\text { saponina }}{\text { peso }} \text { fresco }=\frac{0,646 x \text { (altura de espuma después de } 30 \text { seg en cms })-0,001}{\text { peso de la muestra en gramos }} \\
\% \text { de saponinas }=\frac{0,646 \mathrm{x}(\text { altura de espuma después de } 30 \text { seg en cms })-0,104}{(\text { peso de la muestra en gramos }) \times 10}
\end{gathered}
$$


6 fases fenológicas y etapa reproductiva con 7 fases, estos resultados concuerdan con quienes afirman que la quinua tiene 12 fases fenológicas, sin incluir la germinación [7]. Las etapas y fases determinadas en el campo se describen a continuación: etapa vegetativa:

Germinación de la semilla (V0). Emisión de la radícula, la cual ocurre máximo a las 24 h después de humedecer la semilla (Figura 2).

Emergencia (V1). Cuando la plántula rompe la superficie del suelo (Figura 3).

Aparición hojas verdaderas (V2). Cuando fuera de las hojas cotiledonales, aparece la primera hoja verdadera extendida (Figura 4).

Cuatro hojas verdaderas (V3). Cuando se observan dos pares de hojas verdaderas extendidas y las hojas cotiledonales aún se conservan verdes (Figura 5).

Seis hojas verdaderas (V4). Cuando se observan tres pares de hojas verdaderas extendidas y las hojas primarias se tornan de color amarillento por senescencia (Figura 6).

Ramificación (V5). Cuando se observan cuatro pares de hojas verdaderas extendidas con presencia de hojas axilares hasta el tercer nudo, las hojas primarias se han caído y dejado cicatriz en el tallo, también se nota presencia de inflorescencia protegida por las hojas sin dejar al descubierto la panoja (Figura 7).

Figura 2. Germinación ecotipos de quinua.

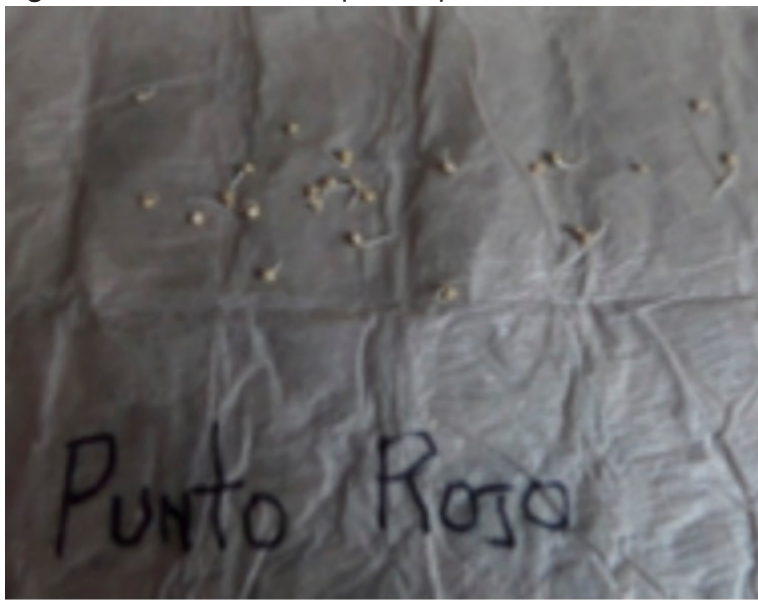

Figura 3. Emergencia de ecotipos de quinua.

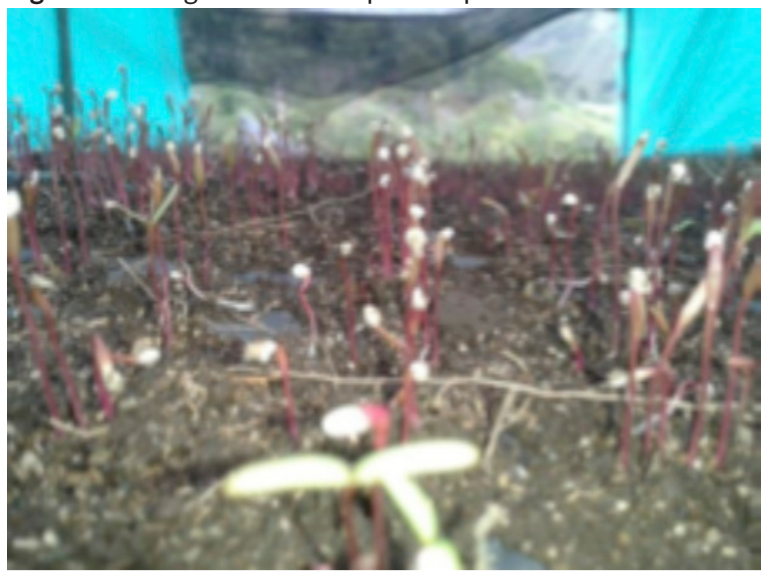

Figura 4. Aparición hojas verdaderas en ecotipos de quinua.

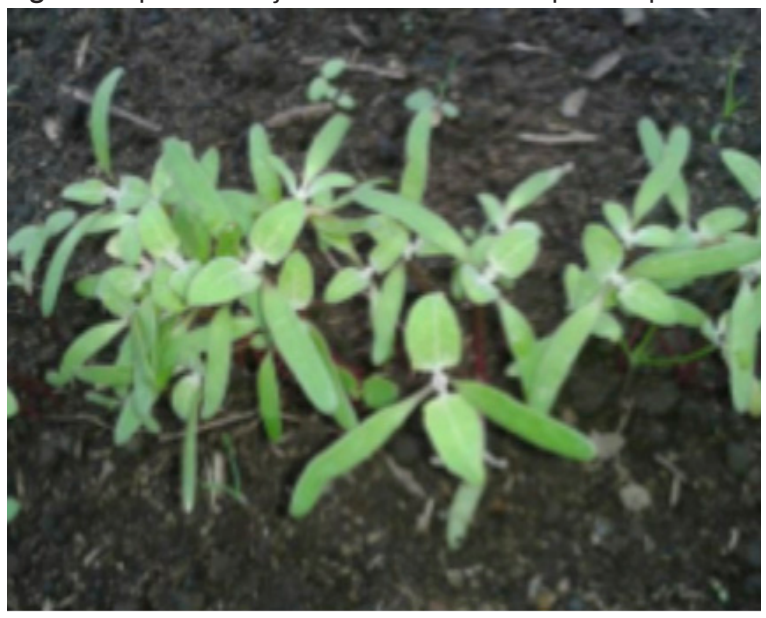

Figura 5. Aparición de hojas verdaderas en ecotipos de quinua.

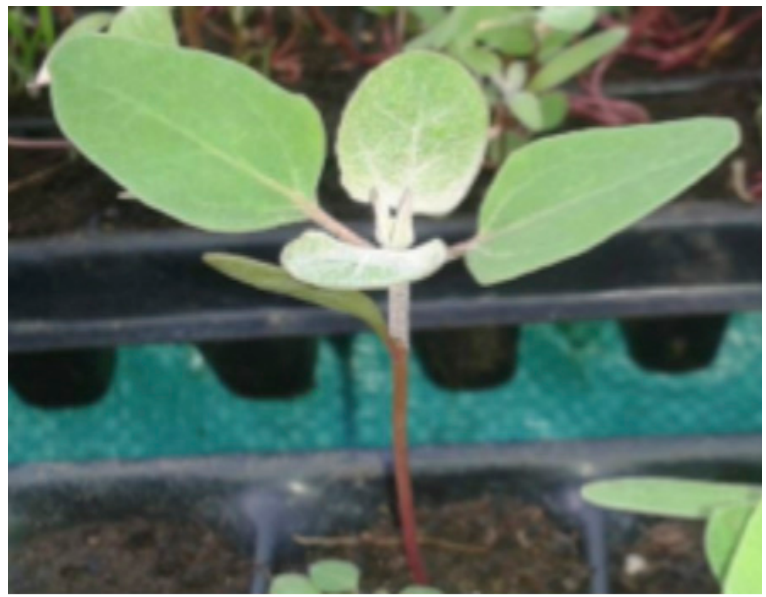


Figura 6. Estado de ecotipos de quinua con 3 hojas verdaderas.

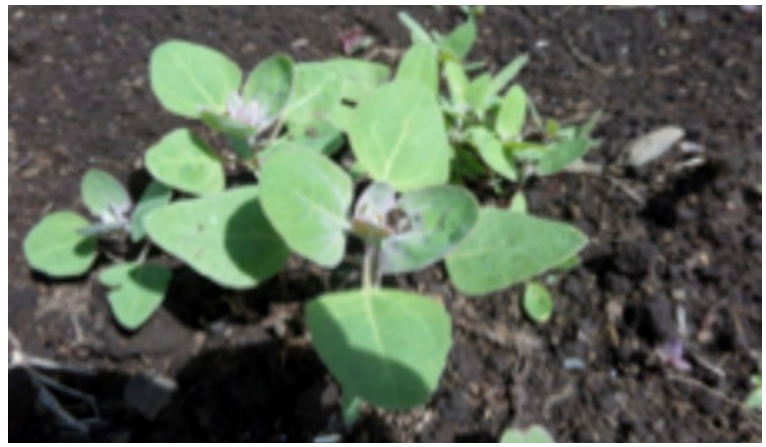

Figura 7. Estado de ecotipos de quinua con 4 hojas verdaderas extendidas.

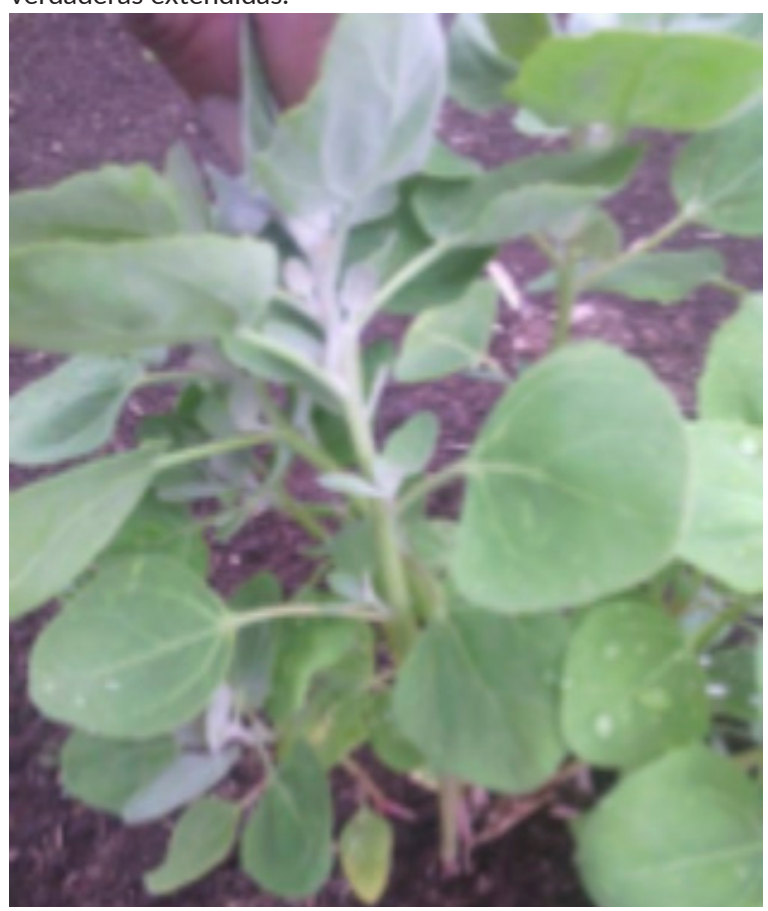

La etapa reproductiva consta de 7 fases, las cuales se describen así:

Inicio de formación de panoja (R6). Cuando del ápice de la planta la emergencia de la inflorescencia es visible y se observa alrededor aglomeración de hojas pequeñas, las cuales van cubriendo la panoja (Figura 8).

Formación de panoja (R7). Cuando se observa claramente la inflorescencia por encima de las hojas, dejando ver claramente los glomérulos que la conforman; además se puede observar en los glomérulos de la base los botones florales individuales (Figura 9).
Inicio de floración (R8). Cuando la flor hermafrodita apical se abre mostrando los estambres separados, se puede notar en los glomérulos las anteras protegidas por el perigonio de un color verde limón (Figura 10).

Floración (R9). Cuando el 50\% de las flores de la inflorescencia se encuentran abiertas y la planta comienza a eliminar las hojas inferiores (Figura 11).

Grano lechoso (R10). Cuando los frutos que se encuentran en los glomérulos de la panoja, al ser presionados explotan y dejan salir un líquido lechoso (Figura 12).

Grano pastoso (R11). Cuando los frutos al ser presionados presentan una consistencia pastosa de color blanco (Figura 13).

Madurez fisiológica y madurez de cosecha (R12) cuando al presionar el grano formado con las uñas,

Figura 8. Inicio de formación de panoja en ecotipos de quinua.

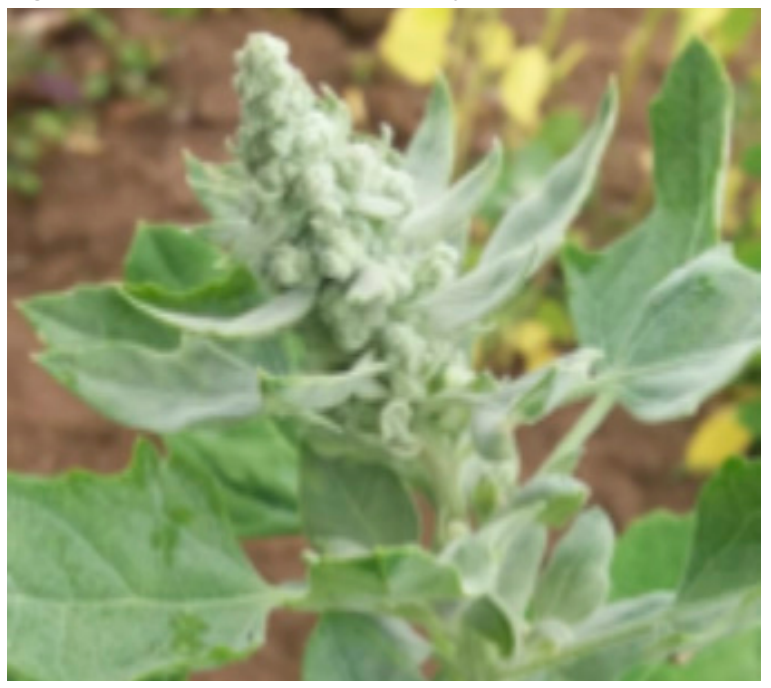

Figura 9. Formación de panoja en ecotipos de quinua.

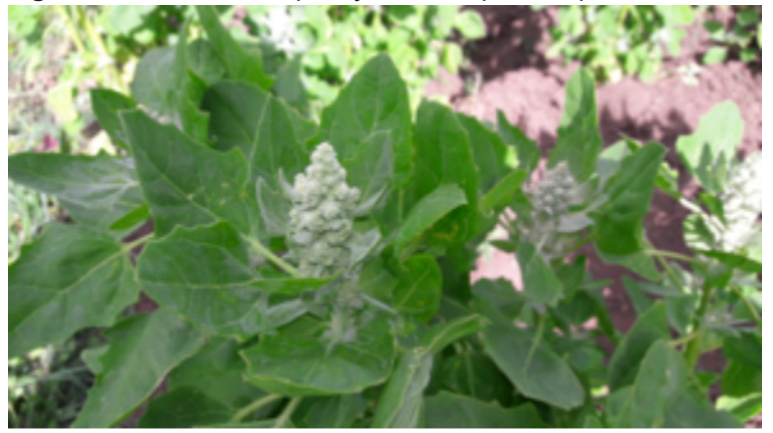


presenta resistencia a la penetración, el contenido de humedad del grano varía de 14 a $16 \%$, en esta etapa inicia el amarillamiento completo de la planta y hay gran defoliación (Figura 14).

Figura 10. Inicio de floración en ecotipos de quinua.

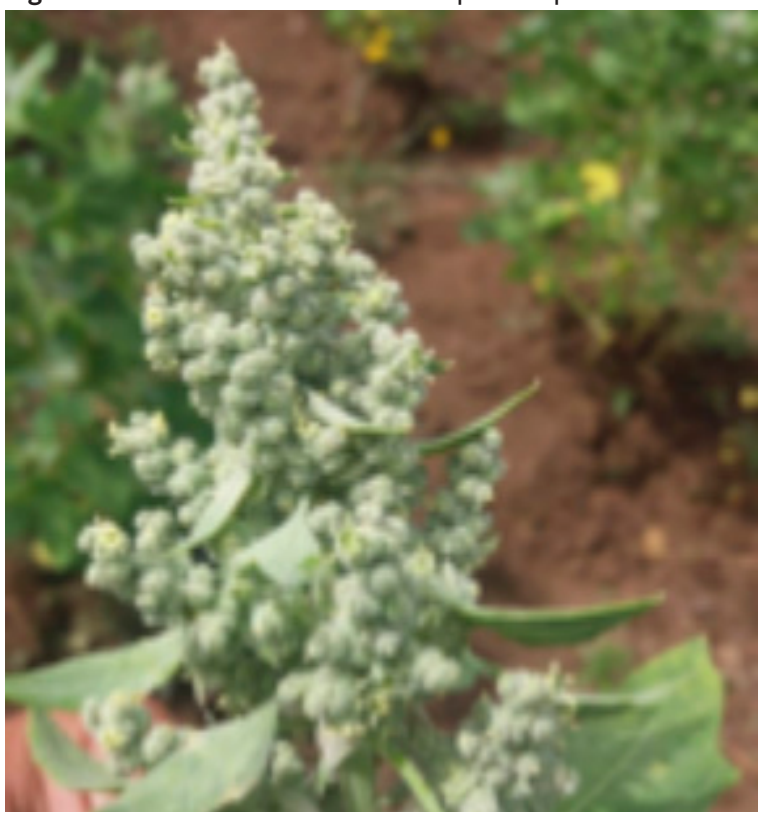

Figura 11. Floración en ecotipos de quinua

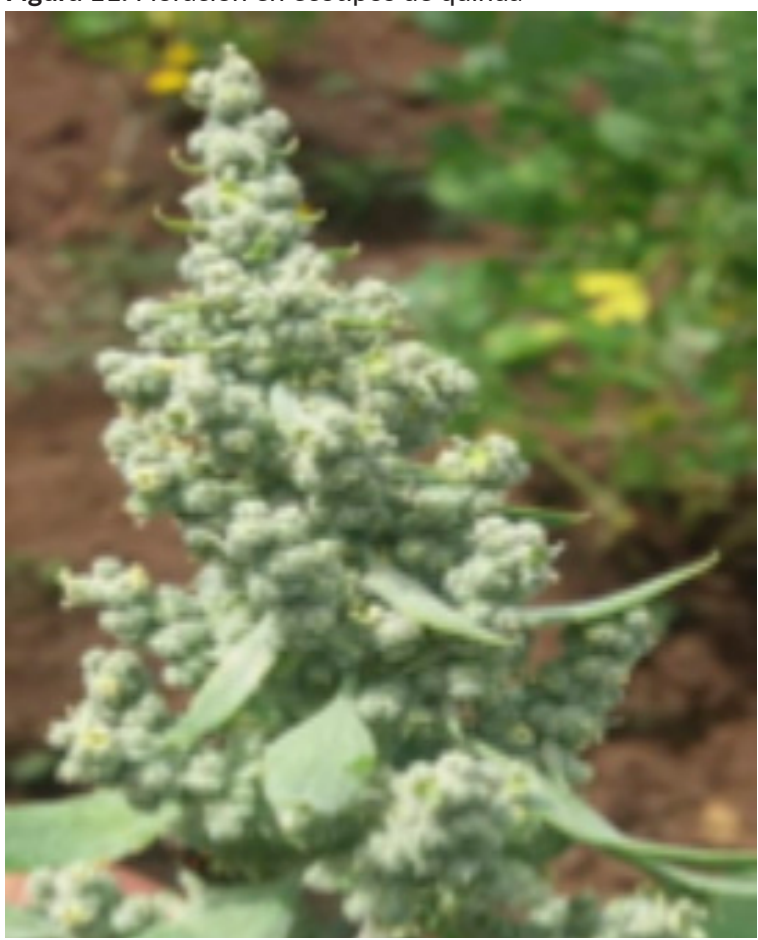

Figura 12. Fase grano lechoso en ecotipos de quinua.

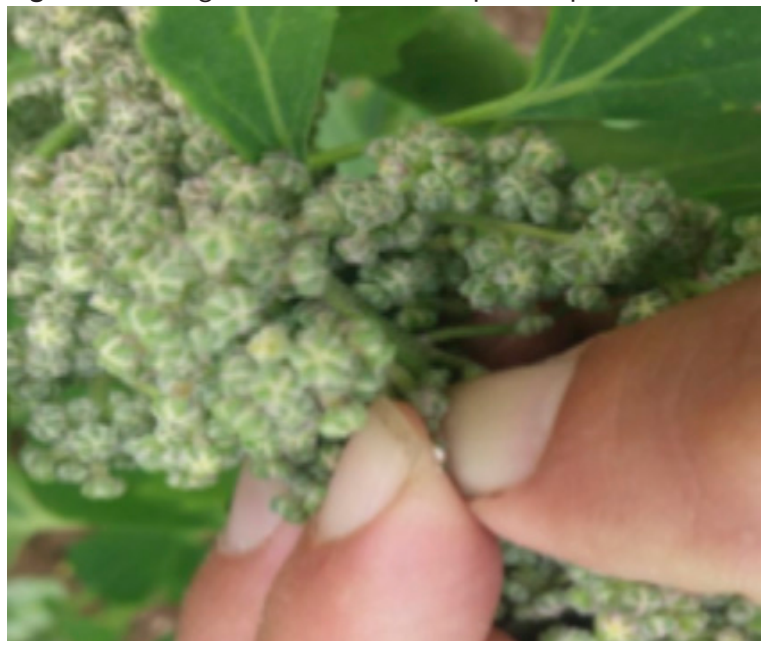

Figura 13. Fase grano pastoso en ecotipos de quinua.

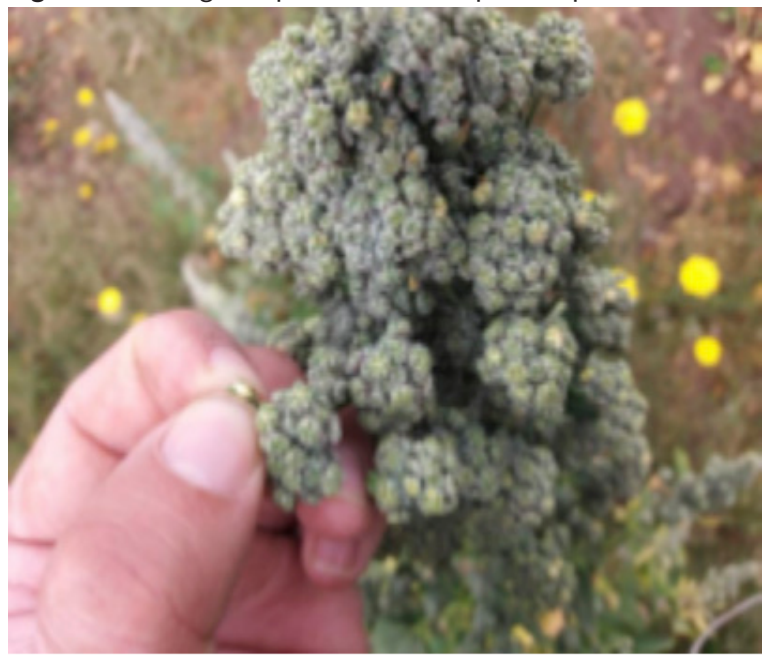

Figura 14. Madurez fisiológica en ecotipos de quinua.

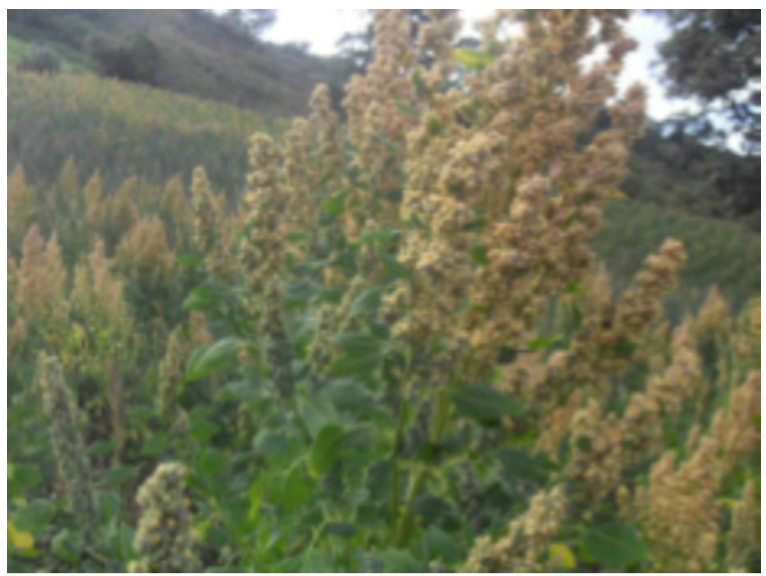


El porcentaje de germinación de semilla fue de $100 \%$, Punto Rojo, Tunkahuan y Aurora lograron este valor a las $24 \mathrm{~h}$ después de siembra, mientras Blanca de Jericó a las $72 \mathrm{~h}$ después de siembra, lo cual se puede deber al espesor de la testa o tamaño del grano [7]., demostrando que la semilla es de muy buena calidad germinativa.

La duración del ciclo fenológico entre los cuatro ecotipos estuvo entre 154,5 y 213,9 días (cuadro 1 ).

El análisis de varianza $(P \leq 0,05)$ (cuadro 2), detectó diferencias estadísticamente significativas en todas las fases de la etapa reproductiva. A partir de la R6, Aurora, acelera su ciclo reproductivo mostrando al final el ciclo más corto de cultivo (154 días), seguido de Tunkahuan con 178 días, considerándolos como semiprecoz y semitardío respectivamente, de acuerdo a la clasificación de semiprecoz para materiales cuyo ciclo está entre 130 y 150 días y semitardío para materiales con ciclo de 150 a 180 días, los otros dos materiales son considerados tardíos [8].

Se afirma que la quinua tarda entre 7 y 10 días para emerger [9], en esta investigación fue máximo de 3 días para todos los ecotipos evaluados (cuadro 1), igualmente reportan diferencias significativas en días a ramificación, lo cual no coincide con esta investigación.

La duración del ciclo productivo está directamente relacionado con las condiciones ambientales, especialmente temperatura y precipitación [7]. La temperatura mínima durante el desarrollo de la investigación osciló entre 4 y $8^{\circ} \mathrm{C}$, la máxima entre 10 y $19^{\circ} \mathrm{C}$ con temperatura promedio entre 7 y $12,5^{\circ} \mathrm{C}$. La temperatura estuvo en el promedio óptimo para quinua entre 6 y $17^{\circ} \mathrm{C}$ [6] lo mismo que la precipitación que alcanzó un máximo de $494 \mathrm{~mm}$ durante el ciclo, que se acerca al máximo aceptado por el cultivo que oscila entre 250 y $500 \mathrm{~mm}$ para cubrir los requerimientos [10]. Se destaca que en los primeros días hubo agua suficiente para germinación y emergencia y hasta el día 30 se tuvieron casi $100 \mathrm{~mm}$ de precipitación, lo cual influyo en el buen desarrollo del cultivo, pero incidió en el desarrollo de enfermedades fungosas que atacaron las plántulas.

Debido al fenómeno del niño durante la investigación se observó plasticidad en el desarrollo de las plantas al presentar ritmos variables de crecimiento, crecieron cuando hubo humedad y detuvieron su crecimiento durante el déficit; pero hubo pronta recuperación después del período de sequía; se observó tolerancia a la desecación y a la deshidratación de los tejidos, al calor evitando escaldaduras y quemaduras de las hojas por los cristales de oxalato de calcio y asincronía en la floración de la panoja, coincidiendo con [8]. Una parte de las flores en los glomérulos estaba en pre antesis, otra en antesis y las demás en formación o llenado del grano. Además, se presentó germinación de las semillas por las altas presiones osmóticas [11], lo cual se comprobó al observar la germinación del grano en la propia panoja, cuando iniciaron las lluvias.

Se ha encontrado que la quinua mantiene la turgencia y cumple las principales funciones vitales, a pesar de la reducción del potencial hídrico, debido a la acumulación activa de solutos en respuesta al déficit de humedad, pre-acondicionamiento [11].

Con el fin de determinar cuáles ecotipos se diferenciaban se realizó prueba de promedios de Duncan, la cual permitió concluir que Aurora se diferenciaba significativamente de los otros tres ecotipos y que

Cuadro 1. Resultados promedios de la fenología de cuatro ecotipos de quinua bajo condiciones del municipio de Puracé, resguardo indígena de Paletará, Cauca.

\begin{tabular}{|c|c|c|c|c|c|c|c|c|c|c|c|c|c|c|}
\hline \multirow{3}{*}{ Ecotipo } & & \multicolumn{6}{|c|}{ Etapa vegetativa } & \multicolumn{7}{|c|}{ Etapa reproductiva } \\
\hline & & Vo & V1 & V2 & V3 & V4 & V5 & R6 & R7 & R8 & R9 & R10 & R11 & R12 \\
\hline & $*$ & \multicolumn{13}{|c|}{ Días después de siembra } \\
\hline Blanca de Jericó & 1 & 2 & 3,0 & 27,3 & 33,0 & 38,7 & 63,2 & 96,2 & 130,4 & 135,4 & 148,3 & 185,2 & 200,3 & 213,9 \\
\hline Punto Rojo & 2 & 1 & 3,0 & 27,4 & 33,1 & 36,7 & 55,8 & 95,9 & 135,1 & 140,2 & 151,9 & 185,4 & 202,1 & 212,2 \\
\hline Tunkahuan & 3 & 1 & 3,0 & 27,4 & 32,8 & 38,8 & 62,2 & 87,2 & 120,2 & 122,8 & 129,9 & 146,0 & 162,7 & 178,7 \\
\hline Aurora & 4 & 1 & 3,0 & 27,0 & 33,0 & 38,8 & 62,6 & 61,4 & 89,7 & 94,9 & 102,1 & 126,2 & 136,1 & 154,5 \\
\hline
\end{tabular}

*No. del ecotipo. V1: Emergencia. V2: aparición hojas verdaderas. V3: Cuatro hojas verdaderas. V4: Seis hojas verdaderas. V5: Ramificación. R6: Inicio de formación de panoja. R7: Formación de panoja. R8: inicio de floración. R9: Floración. R10: Grano Lechoso. R11: Grano pastoso. R12: Madurez fisiológica 
Cuadro 2. Análisis de varianza para las fases reproductivas de 4 ecotipos de quinua bajo condiciones de Puracé, Cauca.

\begin{tabular}{|c|c|c|c|c|c|c|}
\hline Fuente de variación & & Suma de cuadrados & df & Cuadrados medios & $\mathbf{F}$ & Sig. \\
\hline \multirow{3}{*}{ Inicio panoja } & Entre ecotipos & 14403,1 & 3,0 & 4801,0 & 66,7 & $*$ \\
\hline & Dentro de cada ecotipo & 4824,8 & 67,0 & 72,0 & & \\
\hline & Total & 19227,9 & 70,0 & & & \\
\hline \multirow{3}{*}{ Formación de panoja } & Entre ecotipos & 22517,4 & 3,0 & 7505,8 & 44,8 & $*$ \\
\hline & Dentro de cada ecotipo & 11386,6 & 68,0 & 167,4 & & \\
\hline & Total & 33904,0 & 71,0 & & & \\
\hline \multirow{3}{*}{ Inicio flor } & Entre ecotipos & 22284,6 & 3,0 & 7428,2 & 47,2 & $*$ \\
\hline & Dentro de cada ecotipo & 10693,1 & 68,0 & 157,3 & & \\
\hline & Total & 32977,7 & 71,0 & & & \\
\hline \multirow{3}{*}{ Floración } & Entre ecotipos & 28040,2 & 3,0 & 9346,7 & 50,5 & $*$ \\
\hline & Dentro de cada ecotipo & 12588,5 & 68,0 & 185,1 & & \\
\hline & Total & 40628,7 & 71,0 & & & \\
\hline \multirow{3}{*}{ grano lechoso } & Entre ecotipos & 47102,4 & 3,0 & 15700,8 & 65,0 & $*$ \\
\hline & Dentro de cada ecotipo & 16431,3 & 68,0 & 241,6 & & \\
\hline & Total & 63533,7 & 71,0 & & & \\
\hline \multirow{3}{*}{ grano pastoso } & Entre ecotipos & 54710,5 & 3,0 & 18236,8 & 63,0 & $*$ \\
\hline & Dentro de cada ecotipo & 19677,2 & 68,0 & 289,4 & & \\
\hline & Total & 74387,7 & 71,0 & & & \\
\hline \multirow{3}{*}{ Madurez fisiológica } & Entre ecotipos & 44155,3 & 3,0 & 14718,4 & 67,5 & $*$ \\
\hline & Dentro de cada ecotipo & 14823,4 & 68,0 & 218,0 & & \\
\hline & Total & 58978,7 & 71,0 & & & \\
\hline \multirow{3}{*}{ Rendimiento } & Entre ecotipos & 138300000 & 5 & 27650622,2 & 7,41 & $*$ \\
\hline & Dentro de cada ecotipo & 44768055 & 12 & 3730671,25 & & \\
\hline & Total & 183000000 & 17 & & & \\
\hline
\end{tabular}

era el material más precoz en todas las fases reproductivas, seguido de Tunkahuan. (Cuadro 3).

En cuanto a rendimiento el mejor ecotipo fue Tunkahuan (cuadro 4) y el análisis de varianza detecto diferencias estadísticamente significativas entre ecotipos para rendimiento (cuadro 2), la prueba de Duncan (cuadro 3) clasifico a Tunkahuan y Aurora en el mismo grupo.

\section{Determinación del contenido de saponinas}

No se detectaron diferencias entre las repeticiones (cuadro 5). Blanca de Jericó y Aurora tuvieron contenidos de saponinas similares del $0,0012 \%$, mientras Punto rojo y Tunkahuan tuvieron 0,0050\%. Al evaluar el banco de germoplasma de la Estación Experimental Patacamaya en Bolivia, encontraron que las variedades amargas tuvieron concentración de saponinas superiores al 0,05\% y granos grandes y las accesiones dulces presentaron concentración de saponinas igual o menor a 0,05 [12].

Se reporta que el contenido de saponina en la quinua es variable y depende aparentemente de un grupo de genes en la planta, estimando contenidos de saponinas en algunos ecotipos de quinua los cuales estuvieron en un rango entre $0,0 \%$ y $1,2 \%$ [9]. Se ha demostrado que las variedades de quinua Sajama (1,7 $\%)$ y blanca $(1,9 \%)$ presentan menor concentración de saponinas que las variedades amarillas $(2,3 \%)$ y colorada (2,8\%). Estos valores se obtuvieron después de lavar la quinua a temperatura de $50^{\circ} \mathrm{C}$, donde se removió un 75 a $80 \%$ de la saponina [13]. Las saponinas de la quinua son glucósidos triterpenoidales, localizadas en el pericarpio de las semillas y solubles en metanol y agua [14].

Se dice que el nivel máximo aceptable de saponina en la quinua para consumo humano oscila entre 0,06 y $0,12 \%$ [9]. Esto concuerda con los resultados de pruebas sensoriales realizadas en la Universidad de Ambato, Ecuador, en donde se determinó que el límite máximo de aceptación del contenido de saponina en el grano cocido, fue de 0,1\% [15].Lo anterior demuestra que los ecotipos evaluados están en el rango aceptado para consumo humano. 
Cuadro 3. Prueba de Duncan ( $P \leq 0,05)$, para las fases reproductivas de 4 ecotipos de quinua.

\begin{tabular}{|c|c|c|c|c|}
\hline \multicolumn{5}{|c|}{ Inicio Panoja } \\
\hline \multirow{2}{*}{ ECOTIPO } & \multirow{2}{*}{$\mathrm{N}$} & \multicolumn{3}{|c|}{ Subgrupos para alpha $=0.05$} \\
\hline & & 1 & 2 & 3 \\
\hline 4 & 18 & 61,39 & & \\
\hline 3 & 18 & & 87,22 & \\
\hline 2 & 18 & & & 95,94 \\
\hline 1 & 18 & & & 96,17 \\
\hline \multicolumn{5}{|c|}{ Formación de Panoja } \\
\hline 4 & 18 & 89,67 & & \\
\hline 3 & 18 & & 120,17 & \\
\hline 1 & 18 & & 130,39 & 130,39 \\
\hline 2 & 18 & & & 135,11 \\
\hline \multicolumn{5}{|c|}{ Inicio Flor } \\
\hline 4 & 18 & 94,89 & & \\
\hline 3 & 18 & & 122,83 & \\
\hline 1 & 18 & & & 135,39 \\
\hline 2 & 18 & & & 140,17 \\
\hline \multicolumn{5}{|c|}{ Floración } \\
\hline 4 & 18 & 102,11 & & \\
\hline 3 & 18 & & 129,89 & \\
\hline 1 & 18 & & & 148,33 \\
\hline 2 & 18 & & & 151,94 \\
\hline \multicolumn{5}{|c|}{ Grano Lechoso } \\
\hline 4 & 18 & 126,17 & & \\
\hline 3 & 18 & & 146,00 & \\
\hline 1 & 18 & & & 185,17 \\
\hline 2 & 18 & & & 185,39 \\
\hline \multicolumn{5}{|c|}{ Grano Pastoso } \\
\hline 4 & 18 & 136,11 & & \\
\hline 3 & 18 & & 162,72 & \\
\hline 1 & 18 & & & 200,33 \\
\hline 2 & 18 & & & 202,11 \\
\hline \multicolumn{5}{|c|}{ Madurez Fisiológica } \\
\hline 4 & 18 & 154,50 & & \\
\hline 3 & 18 & & 178,67 & \\
\hline 2 & 18 & & & 212,22 \\
\hline 1 & 18 & & & 213,89 \\
\hline \multicolumn{5}{|c|}{ Rendimiento } \\
\hline 2 & 18 & 1730.8 & & \\
\hline 1 & 18 & & 2012.83 & \\
\hline 4 & 18 & & & 4346.14 \\
\hline 3 & 18 & & & 4485.04 \\
\hline
\end{tabular}

1= Blanca de Jericó; 2= Punto Rojo; 3= Tunkahuan y 4= Aurora
Cuadro 4. Promedio de rendimiento para los ecotipos evaluados.

\begin{tabular}{|l|c|}
\hline \multicolumn{1}{|c|}{ Ecotipo } & Rendimiento/ en kg/ha \\
\hline Blanca de Jericó & 2012,83 \\
\hline Punto rojo & 1730,8 \\
\hline Tunkahuan & 4485,04 \\
\hline Aurora & 4346,14 \\
\hline
\end{tabular}

Cuadro 5. Altura de espuma y contenido de saponinas de 4 ecotipos de quinua.

\begin{tabular}{|l|c|c|c|c|}
\hline \multirow{3}{*}{ Ecotipo } & Repet. & $\begin{array}{c}\text { Muestra de } \\
\text { Quinua (g) }\end{array}$ & $\begin{array}{c}\text { Altura de } \\
\text { Espuma } \\
\text { (cms) }\end{array}$ & \% Saponina \\
\hline \multirow{3}{*}{$\begin{array}{l}\text { Blanca } \\
\text { de Jericó }\end{array}$} & 1 & 0,5 & 0,17 & 0,0012 \\
\cline { 2 - 5 } & 2 & 0,5 & 0,17 & 0,0012 \\
\hline \multirow{4}{*}{ Punto rojo } & 3 & 0,5 & 0,17 & 0,0012 \\
\cline { 2 - 5 } & 1 & 0,5 & 0,2 & 0,005 \\
\cline { 2 - 5 } & 2 & 0,5 & 0,3 & 0,005 \\
\hline \multirow{4}{*}{ Tunkahuan } & 2 & 0,5 & 0,2 & 0,005 \\
\cline { 2 - 5 } & 2 & 0,5 & 0,2 & 0,005 \\
\cline { 2 - 5 } & 3 & 0,5 & 0,2 & 0,005 \\
\hline \multirow{3}{*}{ Aurora } & 1 & 0,5 & 0,2 & 0,005 \\
\cline { 2 - 5 } & 2 & 0,5 & 0,17 & 0,0012 \\
\cline { 2 - 5 } & 3 & 0,5 & 0,17 & 0,0012 \\
\hline
\end{tabular}

\section{CONCLUSIONES}

El ciclo fenológico de los 4 ecotipos durante la etapa vegetativa fue similar y solo se detectan diferencias estadísticamente significativas en la etapa reproductiva, presentando el ciclo más corto el ecotipo Aurora, con una duración de 154 días y el más largo Blanca de Jericó con 214 días.

Se recomienda el ecotipo aurora para cultivar en condiciones de Puracé Cauca, como alternativa para rotación con papa, por buen comportamiento agronómico, bajo contenido de saponinas y buen rendimiento, lo que lo hace apto para consumo humano y puede aportar a la seguridad alimentaria regional.

El contenido de saponinas de Blanca de Jericó y Aurora permite clasificarlas como dulces $(0,0012 \%)$ y Punto rojo y Tunkahuan como amargas (0,005\%). 


\section{AGRADECIMIENTOS}

Los autores agradecen a la Universidad del Cauca por el apoyo en la realización de la investigación, a la fundación de profesionales para el desarrollo integral (PRODESIC) y al resguardo indígena de Paletará.

\section{REFERENCIAS}

[1] CURTI, R.N., DE LA VEGA, A.J., ANDRADE, A.J., BRAMARDI, S.J. and BERTERO, H.D. Adaptive responses of quinoa to diverse agro-ecological environments along an altitudinal gradient in North West Argentina. Field Crops Research, 189, 2016, p. 10-18.

[2] ORGANIZACIÓN DE LAS NACIONES UNIDAS PARA LA ALIMENTACIÓN Y LA AGRICULTURA (FAO). Consideraciones sobre el manejo agronómico del cultivo de quinua en el departamento de Nariño: recopilación de experiencias con pequeños productores - proyecto integrado de transferencia de tecnología. Roma (Italia): FAO - Ministerio de agricultura y Desarrollo Rural, 2015, 44 p.

[3] NASCIMENTO, A.C., MOTA, C., COELHO, I., GUEIFÃO, S., SANTOS, M., MATOS, A.S., GIMENEZ, A., LOBO, M., SAMMAN, N. and CASTANHEIRA, I. Characterisation of nutrient profile of quinoa (Chenopodium quinoa), amaranth (Amaranthus caudatus), and purple corn (Zea mays L.) consumed in the North of Argentina: Proximates, minerals and trace elements. Food Chemistry, 148, 2014, p. 420-426.

[4] CURTI, R.N., DE LA VEGA, A.J., ANDRADE, A.J., BRAMARDI, S.J. and BERTERO, H.D. Multi-environmental evaluation for grain yield and its physiological determinants of quinoa genotypes across Northwest Argentina. Field Crops Research, 166, 2014, p. 46-57.

[5] MARCA-VILCA, S., ESPINOZA, P. y POBLETE, V.A. Multiplicación de semilla de variedades y ecotipos de quinua en valle de majes-Arequipa. Journal of high Andean research, 17(3), 2015, p. 355-368.

[6] ALANOCA, C. y MACHACA, E. Caracterización agromorfológica de 10 accesiones y variedades de quinua (Chenopodium quinoa Willd.) en condiciones del Valle Alto de Cochabamba. Revista Científica de Investigación INFO-INIAF, 1(5), 2015, p. 21-29.
[7] VELOZA, R.C., ROMERO, G. y GÓMEZ, J.J. Respuesta morfoagronómica y calidad en proteína de tres accesiones de quinua (Chenopodium quinoa Willd.) en la sabana norte de Bogotá. Revista U.D.C.A Actualidad \& Divulgación Científica, 19(2), 2016, p. 325-332.

[8] FIALLOS-JURADO, J., POLLIER, J., MOSES, T., ARENDT, P, BARRIGA, N., MORILLO, E., ARAHANA, V., TORRES, M., GOOSSENSC, A. and REYES, A.L. Saponin determination, expression analysis and functional characterization of saponin biosynthetic genes in Chenopodium quinoa Leaves. Plant Science, 250, 2016, p. 188-197.

[9] MURPHY, K.S., MATANGUIHAN, J. and WILEY \& SONS, J. Quinoa: Improvement and Sustainable Production. New Jersey (USA): Wiley BlackWell, 2015, 400 p.

[10] ADOLF, V.I., JACOBSEN, S.E. and SHABALA, S. Salt tolerance mechanisms in quinoa (Chenopodium quinoa Willd.). Environmental and Experimental Botany, 92, 2013, p. 43-54.

[11] MUJICA, A., SUQUILANDA, M., CHURA, E., RUIZ, E., LEÓN, A., CUTIPA, S. y PONCE, C. Producción Orgánica de la quinua (Chenopodium quinoa Willd). Puno (Perú): Sociedad Peruana para el Fomento y Competitividad de la Innovación Agraria (FINCAGRO), 56, 2013, p. 59-61

[12] LOZANO, M., TÍCONA, E., CARRASCO, C., FLORES, Y. y YALMANZA, G.R. Cuantificación de saponinas en residuos de quinua real (Chenopodium quinoa Willd). Revista Boliviana de Quimica, 29(2), 2012, p. 131-138.

[13] GUZMÁN, B., TENORIO, R., CRUZ, D.L., ESPINAL, C, ALVARADO, J.A. and MOLLINEDO, P. Saponins from Chenopodium quinoa Willd and Chenopodium pallidicaulea Ellen as biocontrollers of phytopathogen fungi and hemolysis agents. Revista Boliviana de química, 32(1), 2015, p. 8-14

[14] CARRILLO T., W.I., VILCACUNDO, R. y CAR$\mathrm{PIO}, \mathrm{C}$. Compuestos bioactivos derivados de amaranto y quinua. Actualización en Nutrición, 16(1), 2015, p. 18-22.

[15] MORILLO, A.C., CASTRO, M.A. y MORILLO, Y. Caracterización de la diversidad genética de una colección de quinua (Chenopodium quinoa Willd). Revista biotecnología en el sector agropecuario y agroindustrial, 15(2), 2017, p. 49-56. 